\title{
The fixed points of coprime action
}

\author{
By
}

Paul Flavell

\begin{abstract}
Let $P$ be an odd $\pi$-group that acts as a group of automorphisms on the soluble $\pi^{\prime}$-group $G$. We obtain generators for the fixed points of $P$ on $[G, P]$.
\end{abstract}

Let $\pi$ be a set of primes and suppose that the $\pi$-group $P$ acts as a group of automorphisms on the finite $\pi^{\prime}$-group $G$. It is convenient to work in the semidirect product $G P$. An elementary but important consequence of the Schur-Zassenhaus Theorem is

$$
G=C_{G}(P)[G, P] .
$$

Moreover, if $G$ is abelian then $G=C_{G}(P) \times[G, P]$ and in particular, $C_{[G, P]}(P)=1$. In general however, $P$ does have fixed points on $[G, P]$. This certainly happens if $P$ has prime order and $[G, P]$ is not nilpotent. It is the purpose of this paper to obtain generators for $C_{[G, P]}(P)$.

For each $g \in G$ define

$$
[g, P]=\langle[g, \alpha] \mid \alpha \in P\rangle .
$$

Then $[g, P]$ is $P$-invariant but not necessarily $g$-invariant. An elementary argument shows that

$$
\left\langle P, P^{g}\right\rangle=[g, P] P \quad \text { and } \quad[g, P]=\left\langle P, P^{g}\right\rangle \cap G .
$$

Sometimes it is helpful to think of $[g, P]$ in this way. We have

$$
[G, P]=\langle[g, P] \mid g \in G\rangle .
$$

We shall prove the following result.

Theorem A. Let $\pi$ be a set of odd primes and suppose that the $\pi$-group P acts as a group of automorphisms on the soluble finite $\pi^{\prime}$-group $G$. Then

$$
C_{[G, P]}(P)=\left\langle C_{[g, P]}(P) \mid g \in G\right\rangle .
$$

The restriction that $\pi$ consist only of odd primes is essential. Indeed, if $|P|=2$ then $\left\langle P, P^{g}\right\rangle$ is dihedral so $[g, P]$ is inverted by $P$. Thus $C_{[g, P]}(P)=1$ for all $g \in G$.

Conjecture. Theorem A holds for all finite $\pi^{\prime}$-groups $G$. 
In what follows we assume Theorem A to be false and that $G$ is a minimal counterexample. Then $P$ acts non trivially on $G$. Since $G=C_{G}(P)[G, P]$ we have $[G, P]=[[G, P], P]$ so the minimality of $G$ forces

$$
G=[G, P] \text {. }
$$

Let

$$
D=\left\langle C_{[g, P]}(P) \mid g \in G\right\rangle
$$

so that $D$ is a proper normal subgroup of $C_{G}(P)$. Note also that

$$
G \neq[g, P]
$$

for all $g \in G$. Moreover $G$ is nonabelian since otherwise $C_{[G, P]}(P)=1$.

Lemma 1. Let $V \neq 1$ be a proper P-invariant normal subgroup of $G$. Then the following hold.

(a) $C_{G}(P)=D C_{V}(P)$ and $C_{V}(P) \neq D$.

(b) There is a prime $q$ such that $F(G)$ is a q-group.

(c) If $V$ is nilpotent then $V$ is elementary abelian.

(d) $Z(G) \cap D=1$ and $Z(G) \leqq C_{G}(P)$.

Proof. (a) Let $\bar{G}=G / V$. Then as $G=[G, P]$ we have $\bar{G}=[\bar{G}, P]$ and the minimality of $G$ implies that

$$
C_{\bar{G}}(P)=\left\langle C_{[\bar{g}, P]}(P) \mid \bar{g} \in \bar{G}\right\rangle .
$$

If $g \in G$ then $[g, P]$ maps onto $[\bar{g}, P]$ so $C_{[g, P]}(P)$ maps onto $C_{[\bar{g}, P]}(P)$ by $[2$, Theorem 6.2.2(iv), p. 224]. Thus $C_{\bar{G}}(P)=\bar{D}$ whence $C_{G}(P) \leqq D V$ and then $C_{G}(P)=D C_{V}(P)$. This proves (a).

(b) Let $q$ be a prime divisor of $|F(G)|$. Since a Sylow $q$-subgroup of $F(G)$ is normal in $G$, it follows from (a) that $C_{G}(P) / D$ is a nontrivial $q$-group. Applying this argument again, we see that $q$ is the only prime divisor of $|F(G)|$.

(c) Suppose that $\Phi(V)$, the Frattini subgroup of $V$, is nontrivial. From (a) we have $C_{G}(P)=D C_{\Phi(V)}(P)$. Then

$$
V=[V, P] C_{V}(P)=[V, P](D \cap V) C_{\Phi(V)}(P) .
$$

Recall that $[V, P] \triangleq V$ so $[V, P](D \cap V)$ is a subgroup. As $V=[V, P](D \cap V) \Phi(V)$ we deduce that $V=[V, P](D \cap V)$. Thus $C_{V}(P)=C_{[V, P]}(P)(D \cap V)$. The minimality of $G$ implies that $C_{[V, P]}(P) \leqq D$ whence $C_{V}(P) \leqq D$, a contradiction. We deduce that $\Phi(V)=1$, so $V$ is elementary abelian.

(d) Note that $Z(G) \cap D$ and $[Z(G), P]$ are proper $P$-invariant normal subgroups of $G$. Moreover, as $Z(G)$ is abelian we have $C_{[Z(G), P]}(P)=1$. Now apply (a).

\section{Lemma 2. $G$ is not nilpotent.}

Proof. Assume that $G$ is nilpotent. Let $\bar{G}=G / G^{\prime}$. Now $G$ is soluble so $\bar{G} \neq 1$. As $G=[G, P]$ we have $\bar{G}=[\bar{G}, P]$ and as $\bar{G}$ is abelian we have $C_{\bar{G}}(P)=1$ so $C_{G}(P) \leqq G^{\prime}$. Let $g \in G$. The subgroup $G^{\prime}[g, P]$ is $P$-invariant and normal in $G$. Since $G \neq[g, P]$ and 
$G^{\prime} \leqq \Phi(G)$ we have $G^{\prime}[g, P] \neq G$. Lemma 1 (c) implies that $G^{\prime}[g, P]$ is abelian. Now $G=[G, P]$ so we deduce that $G^{\prime} \leqq Z(G)$. Using Lemma 1 (d) it follows that

$$
G^{\prime}=Z(G)=C_{G}(P) .
$$

Let $g \in G$ and $\alpha \in P$. Working in the semidirect product $G P$ we have $\left\langle P, P^{g}\right\rangle=[g, P] P$. By the Schur-Zassenhaus Theorem there exists $h \in[g, P]$ such that $P^{g}=P^{h}$. Let $c=g h^{-1}$ so that $g=c h$. We have $[P, c] \leqq P \cap G=1$ so $c \in C_{G}(P)=Z(G)$. The previous paragraph implies that $[g, P]$ is abelian so since it is $P$-invariant, we see that $h$ commutes with $h^{\alpha}$. Since $c \in C_{G}(P)=Z(G)$ it now follows that

$$
\left[g, g^{\alpha}\right]=1 \text {. }
$$

Since $G^{\prime}=Z(G)=C_{G}(P)$ the maps $x \mapsto[g, x]$ and $x \mapsto[x, g]$ are endomorphisms of $G$ and $\left[x^{\alpha}, y^{\alpha}\right]=[x, y]$ for all $x, y \in G$. Choose $x, y \in G$. Then

$$
\begin{aligned}
1 & =\left[x y,(x y)^{\alpha}\right] \\
& =\left[x y, x^{\alpha}\right]\left[x y, y^{\alpha}\right] \\
& =\left[x, x^{\alpha}\right]\left[y, x^{\alpha}\right]\left[x, y^{\alpha}\right]\left[y, y^{\alpha}\right] \\
& =\left[y, x^{\alpha}\right]\left[x, y^{\alpha}\right] \\
& =\left[x^{\alpha}, y\right]^{-1}\left[x, y^{\alpha}\right] \\
& =\left[x^{\alpha}, y^{-1}\right]\left[x^{\alpha}, y^{\alpha^{2}}\right] \\
& =\left[x^{\alpha}, y^{-1} y^{\alpha^{2}}\right] .
\end{aligned}
$$

It follows that $\left[y, \alpha^{2}\right] \in Z(G)$ and then that $\alpha^{2}$ acts trivially on $\bar{G}$. Since $\alpha^{2}$ acts trivially on $G^{\prime}$ and since $\alpha^{2}$ has order coprime to $G$ it follows that $\alpha^{2}$ acts trivially on $G$. Since $\alpha$ is an arbitrary element of $P$ and since $P$ has odd order we deduce that $P$ acts trivially on $G$, a contradiction. Thus $G$ is not nilpotent.

We have shown that $G \neq F(G)$ so Lemma 1 implies that $F(G)$ is an elementary abelian $q$-group. Let $K$ be the inverse image in $G$ of a minimal $P$-invariant normal subgroup of $G / F(G)$. Then $K$ is $P$-invariant and normal in $G$. Since $G / F(G)$ is soluble, $K / F(G)$ is an elementary abelian $r$-group for some prime $r$. Since $F(G)$ is a $q$-group we have $r \neq q$. By [2, Theorem 2.2.6(i), p. 224], $K$ possesses a $P$-invariant Sylow $r$-subgroup $R$. Then $K=R F(G)$ and $R$ is elementary abelian. The Frattini Argument yields

$$
G=N_{G}(R) F(G) .
$$

Now $K$ is not nilpotent since $F(G)<K$ whence $[F(G), R] \neq 1$. As $G=N_{G}(R) F(G)$ it follows that $[F(G), R]$ is a $P$-invariant normal subgroup of $G$. Let $V$ be a minimal $P$-invariant normal subgroup of $G$ contained in $[F(G), R]$. Since $F(G)$ is abelian we have $C_{[F(G), R]}(R)=1$ whence

$$
C_{V}(R)=1
$$

Lemma 3. $G=R V$.

Proof. We consider $V$ as a $P R$-module over $G F(q)$. Since $[R V, P] \unlhd P R V$ we see that $V \cap[R V, P]$ is a $P R$-submodule. As $P R$ has order coprime to $q$ it follows from Maschke's 
Theorem that $V$ contains a $P R$-submodule $W$ such that

$$
V=W \times(V \cap[R V, P]) .
$$

Now $[W, P] \leqq W \cap(V \cap[R V, P])=1$ so $P$ acts trivially on $W$. Then so also does $[R, P]$. We have

$$
R=[R, P] C_{R}(P)
$$

so as $C_{V}(R)=1$ we see that $C_{W}\left(C_{R}(P)\right)=1$. Consequently

$$
W=\left[W, C_{R}(P)\right] .
$$

Recall that $D \unlhd C_{G}(P)$ so Lemma 1 (a) implies that $C_{G}(P) / D$ is a $q$-group. Since $r \neq q$ it follows that $C_{R}(P) \leqq D$. Since $P$ acts trivially on $W$ we have $W \leqq C_{G}(P)$ whence $W=\left[W, C_{R}(P)\right] \leqq D$.

From $(*)$ we have

$$
C_{V}(P)=W \times\left(V \cap C_{[R V, P]}(P)\right) .
$$

Lemma 1 (a) implies that $C_{V}(P) \neq D$ whence $C_{[R V, P]}(P) \neq D$. The minimality of $G$ forces $G=R V$.

We are now in a position to complete the proof of Theorem A. We will regard $V$ as a $G P$ module over $G F(q)$. Let $\bar{G}=G / V$. Since $G=[G, P]$ we have $\bar{G}=[\bar{G}, P]$. The previous lemma implies that $\bar{G}$ is abelian whence $C_{\bar{G}}(P)=1$ and then $C_{G}(P) \leqq V$. In particular, $C_{R}(P)=1$. Choose $g \in R^{\sharp}$. Now $[g, P] \neq 1$ is a $P$-invariant subgroup of $R, R$ is abelian and $G=R V$. The minimal choice of $R$ implies that $R=[g, P]$.

Let $v \in V$ and consider the $P$-invariant subgroup $[g v, P]$. We have $\overline{[g v, P]}=[\overline{g v}, P]=$ $[\bar{g}, P]=\bar{R}=\bar{G}$ whence

$$
G=[g v, P] V .
$$

Now $G \neq[g v, P]$ and $V$ is a minimal $P$-invariant normal subgroup of $G$. Then $[g v, P] \cap V=1$ and it follows that $[g v, P]$ is a $P$-invariant Sylow $r$-subgroup of $G$. Since $R$ is also a $P$-invariant Sylow $r$-subgroup of $G$, there exists $u \in C_{G}(P)$ such that $[g v, P]^{u}=R$ by [2, Theorem 6.2.2(ii), p. 224].

Working in the semidirect product $G P$, we have

$$
\left\langle P, P^{g}\right\rangle=[g, P] P=R P \quad \text { and } \quad\left\langle P, P^{g \nu}\right\rangle=[g v, P] P .
$$

Then $P^{g v u} \leqq([g v, P] P)^{u}=R P$ and $P^{g} \leqq R P$. Now $C_{G}(P) \leqq V$ so $u \in C_{V}(P)$ and also $v u \in V$. Then

$$
\left[v u, P^{g}\right] \leqq\left\langle P^{g}, P^{g v u}\right\rangle \cap V \leqq P R \cap V=1
$$

so $v u \in C_{V}\left(P^{g}\right)$. We deduce that $V=C_{V}(P) C_{V}\left(P^{g}\right)$ whence

$$
\operatorname{dim}(V) \leqq 2 \operatorname{dim}\left(C_{V}(P)\right) .
$$

Recall that $C_{V}(R)=1$, that $C_{R}(P)=1$ and that $R$ is abelian. The following lemma provides a contradiction and completes the proof of Theorem A. 
Lemma 4. Suppose that $V$ is an $X$-module over a field of characteristic $q$ where $X=R P, R$ is an abelian normal subgroup of $X,(|P|,|R|)=1, R=[R, P], C_{V}(R)=0$ and $X$ is a $q^{\prime}$-group. If $t$ is the smallest prime dividing $|P|$ then

$$
\operatorname{dim}(V) \geqq t \operatorname{dim}\left(C_{V}(P)\right) .
$$

Proof. We may assume that the field is algebraically closed and that $X$ acts irreducibly and faithfully on $V$. Let $V_{1}, \ldots, V_{n}$ be the homogeneous components of $R$, so that $V=V_{1} \oplus \ldots \oplus V_{n}$ and $P$ acts transitively on $\left\{V_{1}, \ldots, V_{n}\right\}$. If $n=1$ then since $R$ is abelian, it is cyclic and acts as scaler transformations on $V$. But then $[R, P]=1$, a contradiction. Thus $n>1$.

For each $i>1$, choose $\alpha_{i} \in P$ such that $V_{1} \alpha_{i}=V_{i}$ and define $\beta_{i}: V_{1} \longrightarrow[V, P]$ by $v_{1} \beta_{i}=v_{1} \alpha_{i}-v_{1}$. Clearly $\operatorname{dim} \operatorname{Im}\left(\beta_{i}\right)=\operatorname{dim}\left(V_{1}\right)$ and it is readily verified that $\operatorname{Im}\left(\beta_{i}\right) \cap\left(\operatorname{Im}\left(\beta_{2}\right)+\cdots+\operatorname{Im}\left(\beta_{i-1}\right)\right)=0$ for all $i \geqq 3$. Consequently,

$$
\operatorname{dim}([V, P]) \geqq(n-1) \operatorname{dim}\left(V_{1}\right) .
$$

Now $\operatorname{dim}(V)=\operatorname{dim}([V, P])+\operatorname{dim}\left(C_{V}(P)\right)$ and $\operatorname{dim}(V)=n \operatorname{dim}\left(V_{1}\right)$ whence

$$
\operatorname{dim}(V) \geqq n \operatorname{dim}\left(C_{V}(P)\right) .
$$

Since $P$ acts transitively on $\left\{V_{1}, \ldots, V_{n}\right\}$ we have that $n$ divides $|P|$ and as $n>1$ we have $n \geqq t$. Hence result

Remark. Theorem A can be used to construct signalizer functors when studying variants of the problem considered in [1].

\section{References}

[1] P. A. Flavell, A characterisation of p-soluble groups. Bull. London Math. Soc. 29, 177-183 (1997).

[2] D. Gorenstein, Finite groups, 2nd edn. New York 1980.

Eingegangen am 15. 4. 1999

Anschrift des Autors:

Paul Flavell

The School of Mathematics and Statistics

The University of Birmingham

Birmingham B15 2TT

United Kingdom 